\title{
Submandibular Approach for Tracheal Intubation in Oro-maxillofacial Surgery: An Initial Experience in a Dental College Hospital in Bangladesh
}

\author{
Md Shahadat Hossain ${ }^{1}$, Hasina Begum², Ismat Ara Haider ${ }^{3}$ Md. Selim Sarker ${ }^{4}$ \\ ${ }^{1}$ Junior consultant, Department of Anaesthesiology, Dhaka Dental College \& Hospital, Dhaka, Bangladesh; \\ ${ }^{2}$ Associate Professor, Department of Anaesthesiology, Dhaka Dental College \& Hospital, Dhaka, \\ Bangladesh; ${ }^{3}$ Associate Professor, Department of Oral \& Maxillofacial Surgery, Dhaka \\ Dental College \& Hospital, Dhaka, Bangladesh; ${ }^{4}$ Junior consultant, Department \\ of Anaesthesiology, Dhaka Dental College \& Hospital, Dhaka, \\ Bangladesh
}

[Received: May 2014; Reviewed: March 2015; Accepted: June 2015; Published: July 2015]

\begin{abstract}
Background: Tracheal intubation in oro-maxillofacial surgery is an important issue during operation. Objective: The purpose of the present study was to demonstrate the feasibility and the reliability of submandibular tracheal intubation as an alternative method for airway management in oro-maxillofacial surgery. Methodology: This clinical trial was conducted in the Department of oromaxillofacial Surgery at Dhaka Dental College and Hospital, Dhaka from January 2010 to June 2011 for a period of one and half year. Patients suffering from painful fractures associated with displaced nasal fracture were selected as study population. Results: A total number of 9 patients were recruited for this study of which 1 was female and 8 were males and the age range was between 16 to 51 years. The technique was found easy and offers a secure airway to the anaesthetist, an optimal operating field and an opportunity to check the dental occlusion with limited morbidity for the patient. In all patients the submandibular tracheal tube was extubated after the operation without complications or difficulties. Accidental dislodgement of the tube to the right main bronchus occurred in one patient while carrying out the procedure. It was rapidly detected and corrected. No other complications were encountered. Conclusion: Submandibular tracheal intubation is a safe, simple and effective technique for upper airway management when both oral and nasal tracheal intubations are not convenient in some oromaxillofacial surgery. [Journal of National Institute of Neurosciences Bangladesh, 2015;1(2): 41-46]
\end{abstract}

Keywords: Submandibular approach; tracheal intubation; oro-maxillofacial surgery

Correspondence: Dr. Md. Shahadat Hossain, Junior consultant, Department of Anaesthesiology, Dhaka Dental College \& Hospital, Dhaka, Bangladesh; Email: shahadat26feb@yahoo.com; Cell no: +8801554236374

Conflict of interest: There is no conflict of interest to any of the authors of this article.

Funding agency: The study was not funded by any authority.

Contribution to authors: MSH, HB and IAH were involved in protocol preparation, data collection and literature search up to manuscript writing. MSS was involved in manuscript preparation and was involved in statistical analysis and manuscript writing. How to cite this article: Hossain MS, Begum H, Haider IA, Sarker MS. Submandibular Approach for Tracheal Intubation in Oro-maxillofacial Surgery: An Initial Experience in a Dental College Hospital in Bangladesh. J Natl Inst Neurosci Bangladesh, 2015;1(2): 41-46

\section{Introduction}

Oromaxillofacial anaesthesia incorporates unique airway problems that require distinctive experience and talented skillful cooperation ${ }^{1}$. The surgeon and the anaesthetist have to operate using good teamwork, in order to attain maximum patient safety with minimal interruption to the surgery.

The standard oral route for tracheal intubation can be unsuitable for some maxillofacial surgeries ${ }^{2}$. It can interrupt the surgical field and can interfere the teeth occlusion frequently needed for the adjustment and fixation of maxillary fractures. The nasal route can be used; however, it can be impossible as a result of deformity or fractures in the nasal bone and it may interrupt some surgical accessibility ${ }^{2-3}$. Nasotracheal intubation is also contraindicated in patients with 
fractures in the cribriform plate of ethmoid which frequently accompany Lefort II and III maxillary fractures because of the potential complications of infection and the possibility of cranial intubation ${ }^{4-6}$. The standard solution in these situations is to perform an elective short term tracheostomy before the operation. Tracheostomy however bears a high risk of complications, especially in children, obese and in patients with an enlarged thyroid gland ${ }^{7}$. An alternative method is to introduce the tracheal tube through a submental $^{8}$ or submandibular ${ }^{9}$ incision which bypasses the surgical area and avoiding the complications of tracheostomy ${ }^{10-14}$. The purpose of the present study was to demonstrate the feasibility and the reliability of submandibular tracheal intubation as an alternative method for airway management in such situations.

\section{Methodology}

This clinical trial was conducted in the Department of oromaxillofacial Surgery at Dhaka Dental College and Hospital, Dhaka from January 2010 to June 2011 for a period of one and half year. Patients suffering from painful fractures associated with displaced nasal fracture were selected as study population. Patients with unsuitable for oral intubation for surgical techniques (teeth occlusion) as well as contraindication for nasal intubation due to nasal bone fractures were selected as study population. After detailed discussion, informed consent was obtained from each patient or legal guardian in case of incompetent patients. Submandibular tracheal intubation was performed on selected patients undergoing surgery under general anaesthesia. Multi-traumatized patient with severe neurological damage or patients presented with major thoracic trauma were excluded from this study; however, tracheostomy was the preferred choice in that case. Preoperative preparations included 8 hours fasting and aspiration prophylaxis with oral ranitidine $150 \mathrm{mg}$ and metoclopramide $10 \mathrm{mg}$ the night before and 2 hours before surgery with sips of water in all cases. The first step in performing the technique was preparing an appropriate size armoured tracheal tube by careful removal of its universal fixed connector to transform it into a removable but fitting connector (Figure 1, 2).

In the operation theatre patient was preoxygenated with $100 \%$ oxygen for three minutes after which anaesthesia was induced with $\mathrm{i} / \mathrm{v}$ fentanyl $1 \mu \mathrm{gm} / \mathrm{kg}$ followed by injection thiopentone $5 \mathrm{mg} / \mathrm{kg}$ intravenously. After induction mask ventilation was checked and found to be adequate. Injection suxamethonium $1.5 \mathrm{mg} / \mathrm{kg}$ was

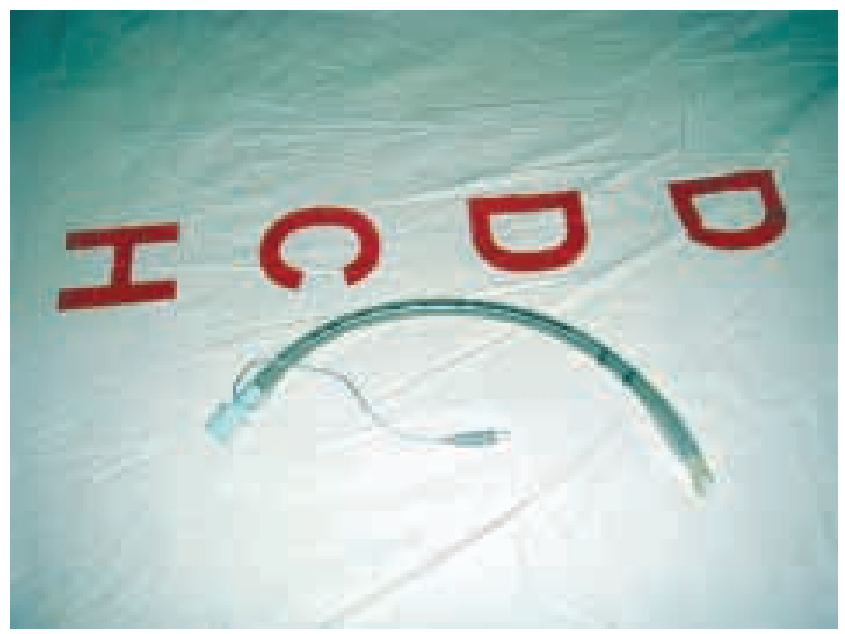

Figure 1: Armoured Tracheal Tube

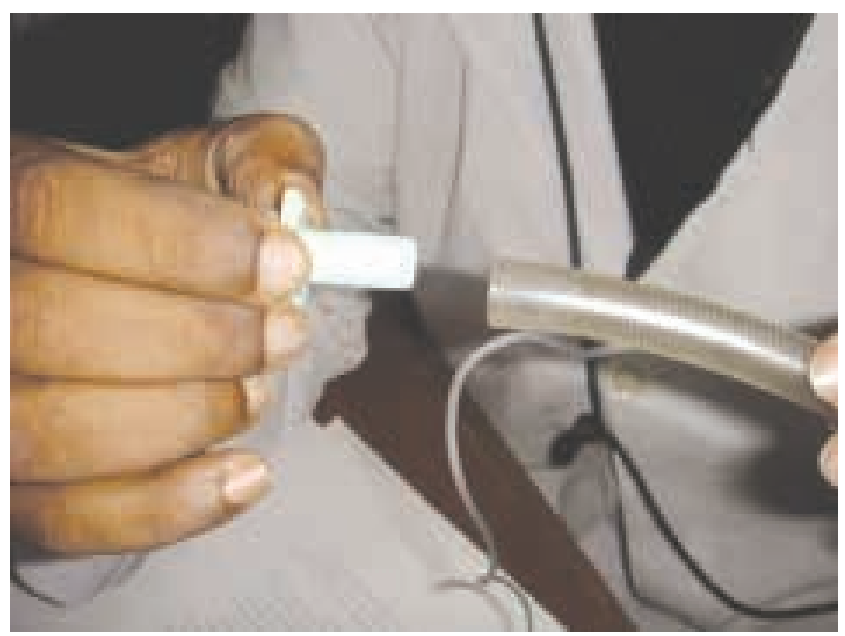

Figure 2: Preparation of an armored tracheal tube by careful removal of its fixed connector to transform it into a removable but fitting connector

administered and ordinary orotracheal intubation using the prepared armoured tube, a temporary draping of the mouth and the submandibular area was carried out. The side of the submandibular area used was dictated by the presence of mandibular fracture. However the right side was usually preferred to permit room for the procedure to be done under vision using a left handed laryngoscope. A $1.5 \mathrm{~cm}$ transverse skin incision was made in the submandibular area about 1 inch below and $1 / 2$ inch anterior to the angle of the mandible. This distance from the lower border of the mandible was to avoid an injury to the mandibular branch of the facial nerve. Using a medium sized curved artery forceps, blunt dissection was carried out through the skin incision in an upwards direction towards the mouth cavities close as possible to the inner side of the mandible. The tissue layer dissected were subcutaneous fat, platysma, investing layer of deep cervical fascia, and the mylohyoid muscle, until the tip of the artery 
forceps tented the mucous membrane of the mouth cavity just medial to the second molar tooth. Incision of the mucous membrane over the tip of the artery forceps enabled the forceps to be introduced into the oral cavity (Figure 3).

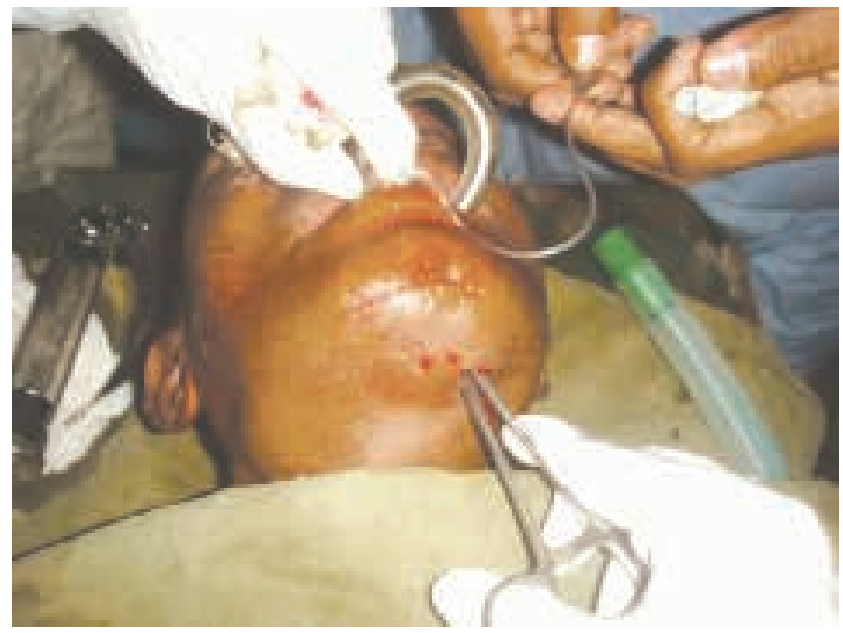

Figure 3: Introducing the tip of the artery forceps in the oral cavity after blunt dissection through the skin incision, as close as possible to the inner side of the mandible

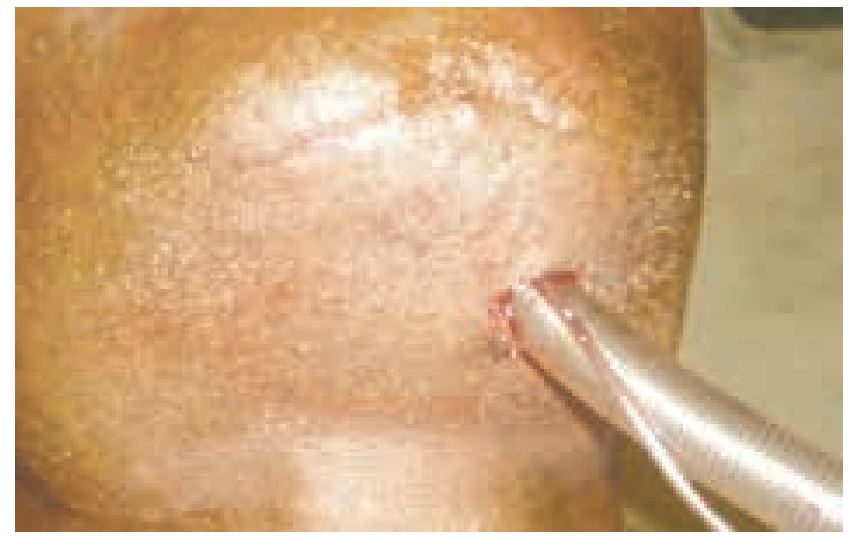

Figure 4: Skin exit site of armoured tracheal tube

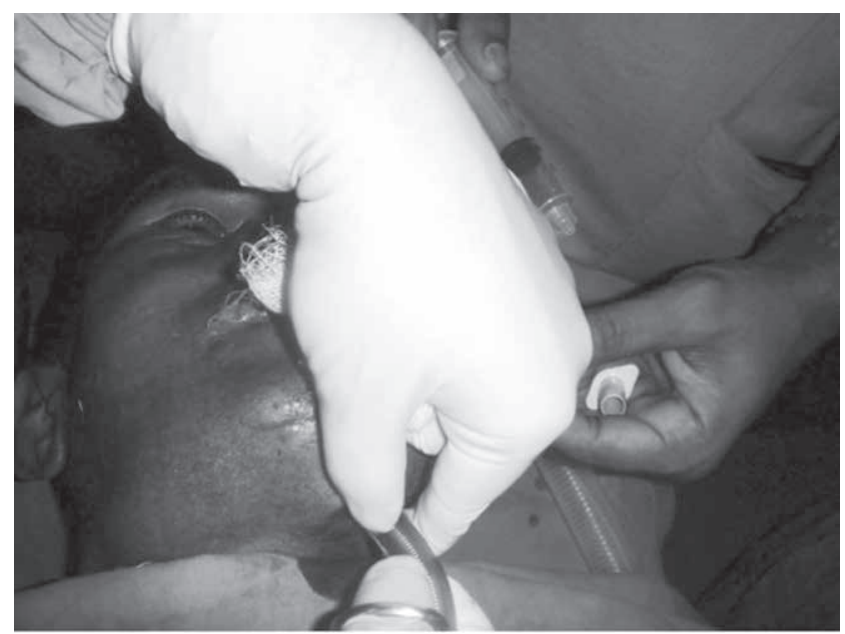

Figure 5: Marking the tube at the skin exit site after checking the tube position with chest auscultation
The end of the cuff inflation tube (pilot tube) was grasped with the tip of the artery forceps and then pulled through the dissected track to come out through the submandibular incision. The patients' lungs were then ventilated with halothane $1 \%$ in oxygen $100 \%$ for 5 minutes and the artery forceps was reintroduced through the skin incision along the same track into the mouth cavity. The tracheal tube was then disconnected from the Bain circuit and its tube connector was removed. Under direct vision using the laryngoscope and with the tube supported in the oropharynx by the tip of the anaesthetist index finger. The end of the tracheal tube was grasped by the tip of the artery forceps and pulled out through the submandibular incision, in the same way as the pilot tube. The tube was supported in the oropharynx through out to prevent accidental extubation or inward pushing of the tube as its end was being pulled out through the track. Any blood that might have intruded into the proximal part of the tube was suctioned immediately and the tube connector was then firmly reattached to the tube before reconnecting the anesthetic circuit. After checking the tube position with chest auscultation a mark made on the tube at the skin exit site (Fig 4, 5). A strong silk stay suture was then made fixing the tube to the skin of the submandibular region. The tube was further secured to the skin with adhesive tape circumferentially applied to the tube over the threads of the stay suture. A pharyngeal pack was then inserted to seal the pharynx from blood and debris during surgery. Care was taken not to damage the pilot balloon. Endotracheal suction could be easily done through this route. At the end of the operation the neuromuscular blocked was reversed with neostigmine and atropine. When patient was fully awake and able to cough and protect his airway then extubation done. After extubation of the tube submandibular skin incision which was then closed by tightening over the previously performed stay suture.

\section{Results}

A total of 221 surgical operations performed under general anaesthesia in the maxillofacial surgical department of Dhaka Dental College and Hospital from January 2010 to June 2011. Submandibular intubation was performed successfully on 9 patients ( 1 female and 8 males). All of the patients were adults with an age ranging from 16 to 51 . 
Table 1: Age and Sex Distribution of Study Population (n=9)

\begin{tabular}{lcc}
\hline Gender & Frequency & Percentage \\
\hline Male & 8 & 88.9 \\
Female & 1 & 11.1 \\
Total & $\mathbf{9}$ & $\mathbf{1 0 0 . 0}$ \\
\hline Age Range & \multicolumn{2}{c}{ 16 to 51 Years }
\end{tabular}

In all the selected patients, oral intubation is not suitable and nasal intubation was contraindicated or impossible. All the patients had panfacial or mid facial fractures. The technique was found to be easy and convenient. No problems were encountered during the procedure of intubation and submandibular exteriorization, and the intraoperative and the post-operative period were uneventful. It provided a secure airway and an uninterrupted surgical access to the nose and to the oral cavity and allowed intraoperative control of dental occlusion. No maxillomandibular fixation was used after operation while the submandibular tube was in place to allow immediate access to the oral airway. However it appeared safe and well tolerated by the patients. There were no problems in the respiratory management of these patients, and endotracheal suctioning could easily be done through the submandibular tube. Extubation in these patients was performed by pulling the tube through the submandibular incision after complete recovery from anaesthesia which was then closed by tightening over the previously performed stay suture. No major complications occurred to our patients and post operative follow up examinations revealed no injury to any of the adjacent structures. The technique was very satisfactory to the surgeon as regards the accessibility to the surgical field and to the anesthetist concerning the safety of the patients' airways. More over as tracheostomy was avoided all the patients and their relatives accepted the technique well and appeared satisfied.

\section{Discussion}

Submental tracheal intubation was first described by Hernandez Altemir in 1986; since then there have been several articles in the literature describing and modifying the technique. However those operation were mostly performed in patients following craniofacial trauma ${ }^{15-16}$. In these patients, nasal intubation was contraindicated because of the possibility of basal skull fracture. The purpose of the present study was to find out a technique for airway management during maxillofacial surgery when both nasal and oral tracheal intubation are deemed unsuitable and to avoid a tracheostomy, especially when long term ventilator support is not required in the postoperative period.

Submandibular intubation is a modification of submental intubation ${ }^{16}$. It is an alternative method for short term tracheostomy when both oral and nasotracheal intubation are contraindicated, impossible or may interrupt the surgical techniques. Altemir's surgical approach for intubation was through a $2 \mathrm{~cm}$ submental incision just medial to the lower border of the mandible, approximately one third of the distance between the symphysis and the angle of the mandible. Potential complications of this approach include damage to adjacent structures, such as submandibular and submaxillary ducts, sublingual gland, and lingual nerve. Several investigators tried Altemirs technique with good success and with no major problems ${ }^{2,15-20}$. However MacInnis and Baig ${ }^{21}$ found it less than satisfactory in their first two patients because of difficult tube passage, bleeding and sublingual gland involvement. Therefore they modified the approach in their next 15 cases to a strict midline submental incision with satisfactory results. Stoll and colleagues ${ }^{9}$ suggested a more posterior approach, in the submandibular area avoiding injury to the important salivary structures. In the present study we used submandibular approach that is considerably far away from the potential complications of submaxillary duct and sublingual gland involvement. In this study it has been found that the technique is easy and effective. It provides an excellent approach to the whole face and the oral cavity without any interruption by the tube. It appeared completely safe as regards the patient's airways. No airway complications or any hypoxic episodes occurred during the technique or the operation.

Surgeons feel comfort with this technique due to adequate surgical access in an uninterrupted operative field inside the mouth. Furthermore the cases of this study involved patients requiring concomitant nasal reconstructive surgery in addition to mandibular or maxillary fixation. By using a submandibular tube it has been avoided intraoperative exchange from nasal to oral tube that can interfere with surgical field, disturb sterilization, and carries some risk of aspiration. Also it can be difficult and disruptive in a patient who recently had repair of fractures. Hypoxia is another potential complication that may occur during the technique if difficulties are encountered during passing the tube through the incision. Moreover, the universal connector of some armoured tubes are designed to be irremovable and may required 
to be cut off and the cut edges of the reinforcing wire may need to be trimmed. Amin et $\mathrm{al}^{18}$ have described the use of capnography during the process of conversion of tube and throughout the surgery to confirm the position of the tube and to serve as a warning tool. Green and Moore ${ }^{22}$ suggested using two tubes, a conventional oro-tracheal tube securing the patients airway whereas a second armoured tube is passed through the incision, from exterior to interior. The second tube is then manipulated with McGill forceps into the oropharynx and then into the trachea just after removal of the first tube. A drawback of this modification is that the cuff of the tube can be damaged during the vigorous manipulation by the McGill forceps ${ }^{2}$. Also to overcome the problem of the irremovable connector, Drolet and colleagues ${ }^{23}$ used a lubricated tube exchanger to replace the submental tracheal tube with a fresh re-enforced armoured one and Amin and Colleagues ${ }^{18}$ used a $100.0 \%$ silicone wire reinforced tube with a removable connector. From this present study it has been agreed with most authors $^{19,24}$ that injury to important structures in the floor of the mouth can be avoided by careful extraperiosteal blunt dissection of the passage as close as possible to the inner side of the mandible and the subperiosteal passage of the tube is not essential especially in the less compared submandibular area. The direction in which this passage is created is another important point of concern. Stranc and Skoracki ${ }^{25}$ reported the development of mucocele while establishing the mucocutaneous track. They use blunt intraoral perforation of the mucous membrane of the mouth and dissected the track from the oral side to the skin. No such complication has been reported in our patients when the track was made as in our series from exterior to interior.

Accidental dislodgement of the tube to the right main bronchus during pulling the tube end through the submandibular track, accidental extubation and inward displacement of the tube were also reported with submandibular intubation while manipulating the mandible during surgery ${ }^{18}$. These complications may be prevented by checking the tube position before fixation and by supporting the tube in the oropharynx by the tip of the anaesthetist's index finger while the tube was being pulled through the track and by proper fixation of the tube.

Postoperative airway protection is often needed after maxillofacial surgical procedures when there is an anticipated risk of airway oedema or haematoma, a delayed return of satisfactory level of consciousness. Removal of the tracheal tube may therefore be delayed until the patient is fully awake; oedema has subsided and a patent and protected airway is guaranteed. In this series, no patient required the submandibular tube to be left in place. On the other hand, rigid plate fixation was used in the patients that rendered immediate postoperative maxillomandibular fixation unnecessary. Therefore postoperative maxillomandibular fixation was not performed in these patients. Using maxillomandibular fixation with the submandibular tube left in place in the post operative period lacks documented experience and is recommended by some authors to be avoided ${ }^{8,15,18}$.

\section{Conclusion}

In conclusion submandibular tracheal intubation is an effective and useful technique for airway control and is associated with only a small risk potential. It can be used as a good alternative to tracheostomy in some maxillofacial surgical patients when tracheal intubation through both oral and nasal routes is not convenient. Therefore by this technique it facilitates the maxillofacial surgery in a less invasive manner by avoiding tracheostomy. Further study is required to define the possible extent and limitations of this strategy. It avoids the need for tracheostomy and its consequent morbidity.

\section{References}

1. Haddock AR, Barnard NA. Maintaining the airway during the treatment of severe facial injuries. Br Dent J 1993:174:56- 7

2. Mak PH, Ooi RG. Submental intubation in a patient with beta-thalassamia major undergoing elective maxillary and mandibular osteotomies. Br J Anaesth 2002;88: 288-91

3. Saravanan P, Arrowsmith JE. Retrograde submental intubation after faciomaxillary trauma. Anesth Analg 2005; 101 :1892-4

4. Zmyslowski WP, Maloney PL. Nasotracheal intubation in the presence of facial fractures. JAMA 1989; 262: 1327-8

5. Muzzi DA, Losasso TJ, Cucchiara RF. Complication from a nasopharyngeal airway in a patient

6. Goodisson DW, Shaw GM, Snape L. Intracranial intubation in patients with maxillofacial injuries associated with base of skull fractures? J Trauma 2001: 50: 363 -6

7. Durbin CG, Jr. Early Complications of tracheostomy. Respir Care $2005: 50: 511-5$

8. Hernandez Altemir F. The submental route of endotracheal intubation: A new technique. J Maxillofac Sur 1986:14: 64-5

9. Stoll P, Galli C, Wachter R, Bahr W. Submandibular endotracheal intubation in panfacial fractures. J Clin Anesth 1994;6:83-6

10. Zeifouni AG, Kost KM. Tracheostomy: a retrospective review of 281 cases. J Otolaryngol 1994; 23:61-6

11. Castling B, Telfer M, Avery BS. Complications of tracheostomy in major head and neck cancer surgery: a retrospective study of 60 consecutive cases. Br J Oral Maxillofacial Surg 1994; 32:3-5

12. Chew JY, Centrell RW. Tracheostomy, complications and their Management. Arch Otolaryngol 1972: 96: 538-45

13. Walker DG. Complications of tracheostomy: their prevention and treatment. J Oral Surg 1973: 31:480-2

14. Stauffer JL, Olson DE, Petty TL. Complications and consequences of endotracheal intubation and tracheostomy. Am J Med 


\section{1;70:65-76}

15. Trepanier CA, Landry PE, Submental Caron G, Paquin R, Lessard MR. Endotracheal intubation: an alternative to tracheostomy in patients with midfacial and panfacial fractures. $\mathrm{J}$ trauma 2000; 48: $235-40$

16. Gordon NC. Tolstunov L. Submental approach to oroendotracheal intubation in patients with midfacial fractures. Oral Sur Oral med Oral Pathol Oral Radiol Endod 1955;79:269-72

17. Paetkau DJ, Stranc MF, Ong BY. Submental orotracheal intubation for maxillofacial surgery. Anesthesiology 2000; 92:912

18. Amin M Dill-Russell P, Manisali M, Lee R, Sinton I. Facial fractures and submental tracheal intubation, Anesthesia 2002;57:1195-9

19. Biglioli F, Mortini P, Goisis M, Bardazzi A Boari N. Submental orotracheal intubation: an alternative to tracheotomy in transfacial cranial base surgery. Skull Base 2003;13:189-95

20. Nyarady Z, Sari F, Olasz L, Nyarady J. Submental endotracheal intubation in concurrent orthognathic surgery: a technical note. J Craniomaxillofac Surg 2006;34:362-5
21. Maclnnis E, Baig M. A modified submental approach for oral endotracheal intubation. Int J Oral Maxillofac Surg 1999;28:344-6

22. Green JD, Moore UJ. A modification of submental intubation. Br J Anaesth 1996;77:789-91

23. Drolet P, Girard M, Poirier J, Grenier Y. Facilitating submental endotracheal intubation with an endotracheal tube exchanger. Anesth Analg 2000; 90:222-3

24. Taglialatela Scafati C, Maio G, Aliberti F. Taglialatela Scafati S, Grimaldi PL. Submento-submandibular intubation: is the subperiosteal passage essential? Experience in 107 consecutive cases. Br J Oral Maxillofac Surg 2006; 44: 12-4

25. Stranc MF, Skoracki R. A complication of submandibular intubation in a panfacial fracture patient. J Craniomaxillofac Surg 2001; 29: 174-6

14. Bigal ME, Liberma JN, Lipton RB. Obesity and migraine. Neurology 2006;66:545-550

15. Mathew NT. Migraine and hypertension. Cephalalgia 1999;19 Suppl 25:17-9 